\title{
Preparation and Characterization of Lecithin-Nano Ni/Fe for Effective Removal of PCB77
}

\author{
Shu Ding, Lin Zhao, Yun Qi, and Qian-qian Lv \\ School of Environmental Science and Engineering, Tianjin University, Tianjin 300072, China \\ Correspondence should be addressed to Lin Zhao; zhaolin@tju.edu.cn
}

Received 5 February 2014; Revised 14 April 2014; Accepted 14 April 2014; Published 30 April 2014

Academic Editor: Fan Dong

Copyright (C) 2014 Shu Ding et al. This is an open access article distributed under the Creative Commons Attribution License, which permits unrestricted use, distribution, and reproduction in any medium, provided the original work is properly cited.

\begin{abstract}
A kind of combined material (named lecithin-nano Ni/Fe) that is composed of lecithin and nanoscale Ni/Fe bimetal was synthesized via microemulsion method. The efficacy of such an original material was tested using $3,3^{\prime}, 4,4^{\prime}$-tetrachlorobiphenyl (PCB77) as target pollutant. A microemulsion system was optimized as template to prepare Ni/Fe nanoparticles, which was followed by an insite loading process with the deposition of lecithin carrier. It was proved by the characterization that subtle Ni/Fe nanoparticles can be uniformly dispersed and closely combined with lecithin carrier. Lecithin was an environmentally compatible biosurfactant that acted as both the component of the microemulsion and the functional material to accumulate organic contaminants. It was expected that the combined material can integrate the functions of lecithin and bimetal. The effectiveness was exhibited through the more rapid and sufficient removal of PCB77 by lecithin-nano Ni/Fe than that by blank carrier. Although requiring further improvement, the constitution of lecithin-nano $\mathrm{Ni} / \mathrm{Fe}$ was a beneficial attempt to acquire the synergistic effect for intensified removal of environmental contaminants. It was promising that the original system and convenient method described in this work will facilitate the development of the organic-inorganic combined materials.
\end{abstract}

\section{Introduction}

Polychlorinated biphenyls (PCBs) are a family comprised of 209 structurally related congeners which are listed as priority chemicals for eventual elimination by 2025 [1]. Their longterm heavy usage together with their high toxicity, chemical stability, long distance transfer, biologic accumulation, and persistence has resulted in their widespread distribution and large environmental risk $[2,3]$. Since the natural degradation of PCBs is very difficult with a conspicuous low speed, PCBs are able to affect the environment and the health of various organisms in a long term. Both the damage of PCBs in the environment and inability of natural degradation at a significant rate highlight the requirement for effective treatment of PCBs. Thus, $3,3^{\prime}, 4,4^{\prime}$-tetrachlorobiphenyl (PCB77), as a coplanar congener (see Figure 1) with high level of toxicity [4], is chosen to be the target compound for the research of contaminant removal.

Recently iron-based bimetallic nanoparticles, as a successful modified material of traditional zerovalent iron, got general attention and further research owing to the obvious advantages including small particle diameter, large specific surface area, and optimal reactive activity $[5,6]$. It had been proved that the iron-based bimetallic nanoparticles were effective in removing chlorinated organic compounds such as PCBs $[7,8]$. While Fe acted as the reductant, Ni could be a proper catalytic metal with good corrosion stability and low cost [9]. But the insufficient activity of $\mathrm{Ni} / \mathrm{Fe}$ nanoparticles required the usage of the expensive catalytic of Pd to remove PCBs, which affected the application potential of the bimetallic material. To reinforce the removal efficacy of $\mathrm{Ni} / \mathrm{Fe}$ nanoparticles, a possible method was to combine them with another material. In this paper, the lecithin carriers were synthesized to load $\mathrm{Ni} / \mathrm{Fe}$ nanoparticles. It was expected that lecithin could contribute to the accumulation of PCB77 around $\mathrm{Ni} / \mathrm{Fe}$ nanoparticles. The great regional concentration of PCB77 will facilitate its interaction with bimetal, which correlated with a good removal efficacy.

The attempts of utilizing lecithin as accumulation material were based on the existing studies about the usage of 


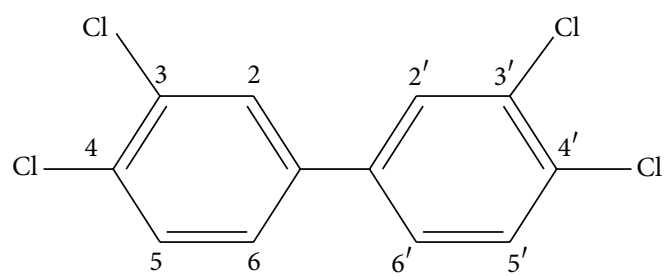

FIgURE 1: Structural formula of $3,3^{\prime}, 4,4^{\prime}$-tetrachlorobiphenyl (PCB77).

surfactant to intensify the treatability of the organic contaminants. Such applications involving the washing process [10$12]$ and the aids for biodegradation $[13,14]$ took advantage of the effects including the solubilization and the emulsification which were offered by surfactant to influence the distribution of the contaminant molecules. The hydrophobic end of the surfactant molecules clustered together inside with the hydrophilic end exposed to the exterior in aqueous solution. This structure can be called the micelle or emulsified droplet. The interior of the micelles or droplets constitutes the dispersed phase wherein the hydrophobic organic contaminant was compatible and accumulated. Therefore the mechanism behind the great apparent solubility and good mobilization of the contaminant was its accumulation in the micelles or droplets.

Surfactants produced from chemically based materials were known as synthetic surfactants and those from biologically based materials were biosurfactants. In general synthetic chemical surfactants were found to persist and exert toxic effects [15-17]. In comparison with synthetic surfactants, biosurfactants had the advantages including being easily produced on renewable resources, possible reuse by regeneration, high specificity, less toxicity, and good biodegradability [18-20]. They were better suited to environmental applications than synthetic ones due to better environmental compatibility and higher activity [21, 22].

Therefore lecithin, as a type of biosurfactant which was low-cost by-product of oil seed industry [23], was a promising accumulation material and expected to be effective in assisting the contaminant removal by $\mathrm{Ni} / \mathrm{Fe}$ bimetal. It was oil soluble and thereby beneficial for the binding of the organic contaminants such as PCBs. Indeed, it had been found that lecithin was effective in interacting with PCBs and assisted the removal process $[24,25]$. However, the combined utilization of lecithin and $\mathrm{Ni} / \mathrm{Fe}$ bimetal had never been tested and may be attractive. Additionally, it was reported that lecithin has been used to generate w/o microemulsion with the addition of coemulsifiers such as short-chained alcohols $[26,27]$. The subtle water droplets that dispersed into the microemulsion were able to dissolve metallic salt and accordingly become the templates for the preparation of $\mathrm{Ni} / \mathrm{Fe}$ nanoparticles. If such a hypothesis was ensured, the synthesis and loading of $\mathrm{Ni} / \mathrm{Fe}$ nanoparticles can be accomplished in one step before the insite generation of lecithin carrier. It was promising to prepare the combined material with convenient technology and to synthesize $\mathrm{Ni} / \mathrm{Fe}$ nanoparticles with advanced character including small particle size, good dispersion, and high activity.

The present work was undertaken to synthesize lecithin carriers loading Ni/Fe bimetal to remove PCB77. The material efficacy was investigated through the removal of PCB77 in 50/ $50(\mathrm{v} / \mathrm{v})$ ethanol/water solution. Lecithin acted not only to accumulate PCB77 but also to constitute a microemulsion system which was the template for the insite generation of $\mathrm{Ni} / \mathrm{Fe}$ nanoparticles with advanced character. The combined material was expected to integrate the function of lecithin and bimetal. To the best of our knowledge, this was the first work in which (a) the microemulsion containing lecithin was produced to be the template for the generation of $\mathrm{Ni} / \mathrm{Fe}$ nanoparticles, (b) lecithin carriers loading $\mathrm{Ni} / \mathrm{Fe}$ bimetal were synthesized, and (c) such a type of combined material was tested to remove PCBs. It was offered by this research that an innovative method to design and prepare functional material for the environmental decontamination. Although requiring further improvement, the method was valuable because the combined material can be prepared conveniently utilizing the stuff including the metallic salt and lecithin which were abundant, low cost, and environmentally compatible.

\section{Materials and Methods}

2.1. Materials. Soybean lecithin powder was purchased from Tianjin Boshuai Industrial \& Trading Co., Ltd. Tween 80 was purchased from Tianjin Guang Fu Chemical Reagents Factory. Analytical grade $\mathrm{FeSO}_{4} \cdot 7 \mathrm{H}_{2} \mathrm{O}$, analytical grade $\mathrm{NiCl}_{2}$. $6 \mathrm{H}_{2} \mathrm{O}$, and superior grade $\mathrm{NaBH}_{4}$ were all purchased from Kemiou Chemical Reagent Co., Ltd. The water used to prepare the solution in the experiment was distilled water. The standard substance of 3,3',4,4' -tetrachlorobiphenyl (PCB77) was provided by AccuStandard Inc. with a purity of $>99.5 \%$. Unless otherwise stated, all other chemicals were of analytical grade and used without further purification prior to use.

2.2. Formulation Studies for the Microemulsion. Five-component system consisting of oil/surfactant (comprised of lecithin and Tween 80)/cosurfactant and water can be described with pseudo ternary phase diagram. The mass ratio of lecithin to Tween 80 was determined to be $0.8: 1$ for the stabilization of the system and the solubilization of more contents of water. According to the preliminary experiments, isopropanol was chosen as the cosurfactant while cyclohexane was chosen to be the oil phase with the advantages that they were abundant, low cost, and relatively low toxic. A mixture of emulsifiers containing equal quantities of the surfactant and the cosurfactant was blended with oil phase at determined mass ratios. The resulting mixture was titrated with distilled water. The volume of water added was recorded while the turbid system turned clear and vice versa. The procedure based on visual inspection was repeated several times at different mass ratios of cyclohexane to emulsifiers. The pseudo ternary phase diagram was constructed by plotting the amounts of oil, surfactant/cosurfactant, and water to identify 
the microemulsion region and thereby define the template formulation for the preparation of $\mathrm{Ni} / \mathrm{Fe}$ nanoparticles.

\subsection{Preparation of Lecithin Carriers Loading Ni/Fe Nanopar-} ticles. After the content of each constituent was determined, the premixed solution of lecithin, Tween 80, isopropanol, and cyclohexane was stirred while a freshly prepared solution of $\mathrm{NaBH}_{4}$ was dropped in the mixture to form the microemulsion template. The microemulsion system was treated using the ultrasound and then transferred into four-necked bottle. Under a nitrogen gas, required amount of the solution of $\mathrm{FeSO}_{4}$ was slowly added into the aforementioned system with high speed stirring at room temperature. Solution color was observed to change to black, which indicated that the ferrous iron was reduced. The generation of $\mathrm{Fe}^{0}$ can be interpreted through the following reaction:

$$
\mathrm{Fe}\left(\mathrm{H}_{2} \mathrm{O}\right)_{6}{ }^{2+}+2 \mathrm{BH}_{4}{ }^{-} \longrightarrow \mathrm{Fe}^{0}+2 \mathrm{~B}(\mathrm{OH})_{3}+7 \mathrm{H}_{2}
$$

Subsequently, after a session for the accomplishment of the reduction, an aqueous solution of $\mathrm{NiCl}_{2} \cdot 6 \mathrm{H}_{2} \mathrm{O}$ was dropped in the system with stirring to react with the wet $\mathrm{Fe}^{0}$ nanoparticles according to the following equation:

$$
\mathrm{Fe}^{0}+\mathrm{Ni}^{2+} \longrightarrow \mathrm{Fe}^{2+}+\mathrm{Ni}^{0}
$$

When the Ni/Fe nanoparticles were synthesized in the microemulsion, the demulsifier of tetrahydro furan was slowly added to acquire the uniform deposition of the lecithin carriers with the $\mathrm{Ni} / \mathrm{Fe}$ nanoparticles loading on them. Asprepared combined material was collected by the high speed centrifugation and rinsed with acetone and ethanol that were both precooled at $4^{\circ} \mathrm{C}$. The products were dried under vacuum before further experiments. Blank lecithin carriers were prepared according to the procedure above except that there was no addition of $\mathrm{NaBH}_{4}$ and metallic salts.

\subsection{Characterization of Lecithin Carriers Loading Ni/Fe Nano-} particles. The morphology of lecithin carriers loading Ni/Fe nanoparticles (in short lecithin-nano $\mathrm{Ni} / \mathrm{Fe}$ ) was investigated using the transmission electron microscopy (TEM, Tecnai $\mathrm{G}^{2}$ F20, Holland) with EDS mapping to study the distribution and the loading ensemble of bimetal component on the carrier. For TEM analysis operated at an acceleration voltage of $200 \mathrm{kV}$, the samples were dispersed by an ultrasonicator in acetone and dropped to a copper grid with a lacy carbon layer. The scanning electron microscopy (SEM, SUPRA 55VP, Germany) was employed to precisely reflect the size, structure, and surface morphology of lecithin-nano $\mathrm{Ni} / \mathrm{Fe}$ for which the samples were dried under vacuum at $50^{\circ} \mathrm{C}$ and then affixed onto adhesive tapes.

The character analyses involving the nitrogen BrunauerEmmett-Teller (BET) surface areas, the X-ray diffraction (XRD), and the Fourier transform infrared spectrophotometer (FT-IR) were performed for lecithin-nano $\mathrm{Ni} / \mathrm{Fe}$ with that for soybean lecithin powder and blank lecithin carriers (nanolecithin) as comparisons. BET surface areas were obtained by nitrogen adsorption using Quadrasorb SI analyzer (Quantachrome, USA). XRD analysis was carried out using XRD diffractometer (Rigaku, Japan) at $40 \mathrm{kV}$ and $100 \mathrm{~mA}$ over the sanning range of $10^{\circ}-90^{\circ} 2 \theta$. Samples for FT-IR (NICOLET6700, USA) measurement were prepared by mixing specimens with $\mathrm{KBr}$ powder and then pressing the mixtures into a sheer slice for analysis in the range of $400-4000 \mathrm{~cm}^{-1}$. An average of 9 scans was collected for each measurement with a resolution of $2 \mathrm{~cm}^{-1}$.

2.5. Removal of PCB77 in Solution. The abilities to remove PCB77, respectively, by lecithin-nano $\mathrm{Ni} / \mathrm{Fe}$ and nanolecithin were determined. The working solution of $50 / 50(\mathrm{v} / \mathrm{v})$ ethanol/water containing about $5 \mu \mathrm{g} \cdot \mathrm{mL}^{-1}$ PCB77 was prepared from the stock solutions. For each sample, about $0.5 \mathrm{~g}$ of the target material was measured and added into a $50 \mathrm{~mL}$ conical flask. Each system was then filled with $40 \mathrm{~mL}$ working solution that had been sufficiently deoxygenated and admixed. The blank system only containing the working solution was simultaneously prepared. The flasks were sealed after their headspace was filled with nitrogen gas and then placed on an orbital shaker. Constant temperature of $30^{\circ} \mathrm{C}$ and rotation speed of $200 \mathrm{rpm}$ were selected as the identical condition for all experiments. At specific reaction time intervals, small amounts of solution were withdrawn to acquire the supernatant phase following centrifugation. For each sample, $600 \mu \mathrm{L}$ of the supernatant phase was transferred into a $2 \mathrm{~mL}$ screw cap bottle and extracted with $\mathrm{n}$-hexane. PCB77 was quantitatively analyzed by GC-ECD (Agilent 7890A GC coupled with a $\mu$-ECD detector) using a DB-1701 column. The injection port was set at $250^{\circ} \mathrm{C}$ and detector temperature was set at $280^{\circ} \mathrm{C}$. A split-splitless injector in the splitless mode was used with $2 \mu \mathrm{L}$ of injection volume for each sample and nitrogen as the carrier gas. The column temperature was programmed as follows: initial temperature at $120^{\circ} \mathrm{C}$ and temperature program rate at $20^{\circ} \mathrm{C} \cdot \mathrm{min}^{-1}$ to $210^{\circ} \mathrm{C}$ and then $25^{\circ} \mathrm{C} \cdot \mathrm{min}^{-1}$ to $260^{\circ} \mathrm{C}$.

\section{Result and Discussion}

3.1. Formulation Studies for the Microemulsion. A phase diagram was required to determine the extent of the monophasic area that corresponds to w/o microemulsion. Before the description, the optimized mass ratio of lecithin to Tween 80 $(0.8: 1)$ and that of surfactant to cosurfactant $(\mathrm{Km}, 1: 1)$ were determined to profit the water incorporation with the fixed composition of the other factors. The w/o area for the fivecomponent systems that consisted of cyclohexane, lecithin, Tween 80, isopropanol, and water was visualized in the pseudo ternary phase diagram (Figure 2). With the narrow feasible region, it was essential to select the appropriate point for preparing the formulation which was consistent with the maximum water incorporation. The existence of sufficient water in w/o microemulsion was beneficial for the good template efficacy through the high loading efficiency and effective dispersion of the metallic components. The optimized mass ratio of cyclohexane/lecithin/Tween 80/isopropanol/water was $73.5: 7: 8.75: 15.75: 27$. The addition of required concentration of $\mathrm{NaBH}_{4}$ was tested and did not obviously affect the stabilization of the w/o microemulsion. 


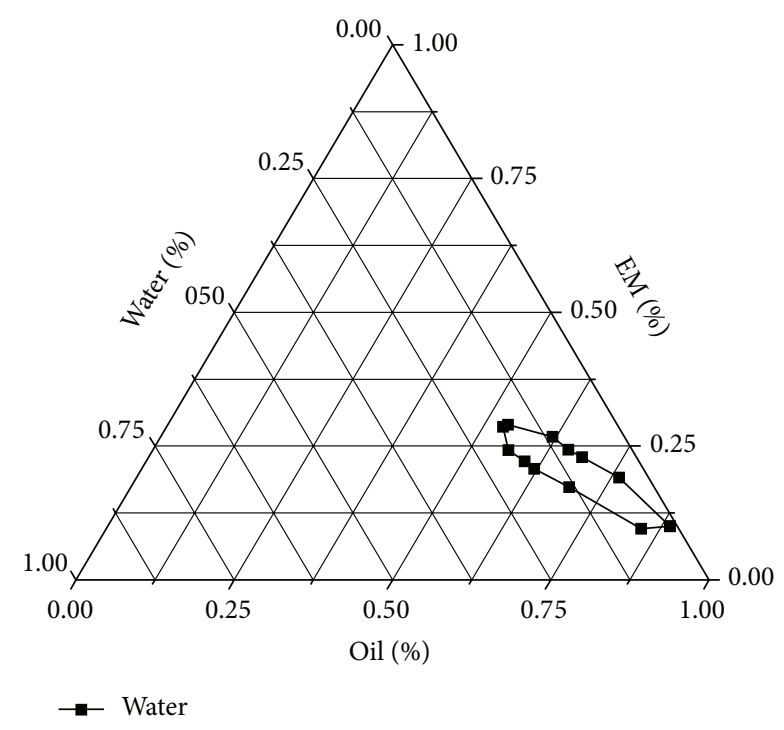

FIGURE 2: Pseudo ternary phase diagram of the w/o microemulsion.

Therefore such a composition of microemulsion was determined to be the template condition for the further synthesis of target materials.

3.2. Morphological Analysis of Lecithin-Nano Ni/Fe. The morphology and state of lecithin-nano $\mathrm{Ni} / \mathrm{Fe}$ were observed by TEM. It was revealed that lots of small spherical nanoparticles were uniformly dispersed on the substrate (Figure 3(a)). The dispersion state of the nanoparticles was consistent with the distribution of the elements of nickel and iron according to the mapping of energy dispersive spectroscopy (EDS) (see Figure 3(b)). Under close inspection of a portion of the carrier, it can be found that the subtle dark-colored nanoparticles had identical size in the range of several nanometers (Figure 3(c)). Based on these results, it was signified that the $\mathrm{Ni} / \mathrm{Fe}$ nanoparticles were reliably synthesized and had a widespread loading state on the carriers. The accurate surface morphology can be obtained from the SEM photogram which presented the dispersion state more clearly (Figure 4). While the lecithin carrier was roughly spherical in overall shape with the size range of $600-700 \mathrm{~nm}$, its surface with abundant interstices was sufficiently covered by the small $\mathrm{Ni} / \mathrm{Fe}$ nanoparticles which were about $10 \mathrm{~nm}$. The structure feature corresponded with the larger surface area and more active sites which will facilitate the effectiveness of lecithinnano Ni/Fe in removing PCB77.

3.3. Analysis of BET Surface Area. The data from the analysis of BET surface area were summarized and displayed in Table 1. It was obvious that the values of the surface area, the pore volume, and the pore diameter for both the lecithinnano $\mathrm{Ni} / \mathrm{Fe}$ and nanolecithin were significantly larger than that for raw material powder, which was attributed to the influence of nanoscale. The slightly larger values for lecithinnano $\mathrm{Ni} / \mathrm{Fe}$ than that for nanolecithin were possibly dedicated
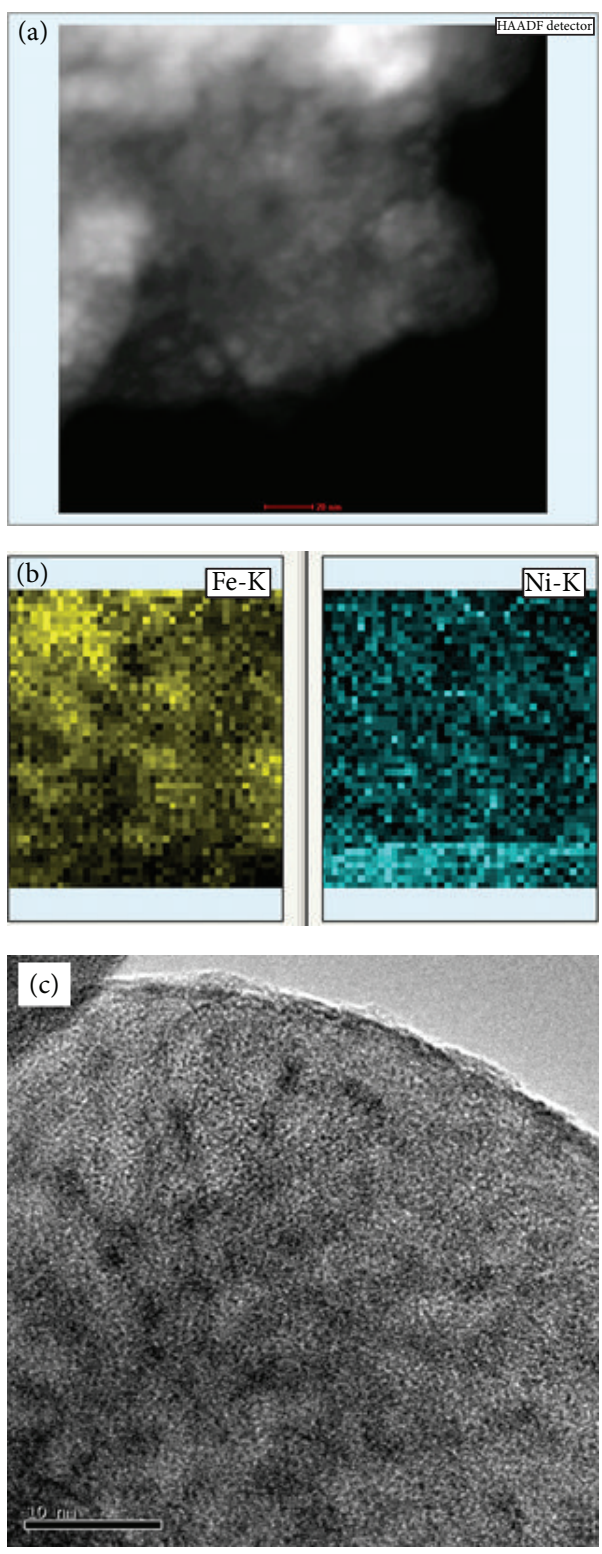

FIGURE 3: TEM micrograph (a) and correlated EDS mapping (b) of lecithin-nano $\mathrm{Ni} / \mathrm{Fe}$, and (c) closely TEM inspection of a portion of the carrier.

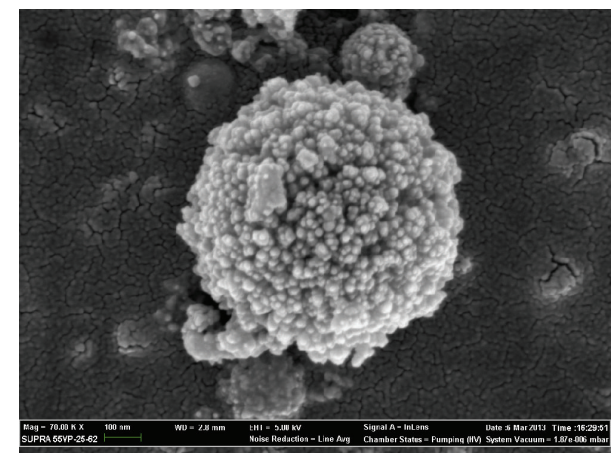

FIGURE 4: SEM micrograph of lecithin-nano Ni/Fe. 
TABLE 1: Data arose from the analysis of BET surface area.

\begin{tabular}{lccc}
\hline Sample & $S_{\mathrm{BET}}\left(\mathrm{m}^{2} / \mathrm{g}\right)$ & $\begin{array}{c}\text { Pore volume } \\
\left(\mathrm{cm}^{3} / \mathrm{g}\right)\end{array}$ & $\begin{array}{c}\text { Pore diameter } \\
(\mathrm{nm})\end{array}$ \\
\hline Lecithin-nano Ni/Fe & 16.24 & 0.0694 & 17.10 \\
Nanolecithin & 15.38 & 0.0542 & 14.10 \\
Lecithin powder & 1.725 & 0.00201 & 4.66 \\
\hline
\end{tabular}

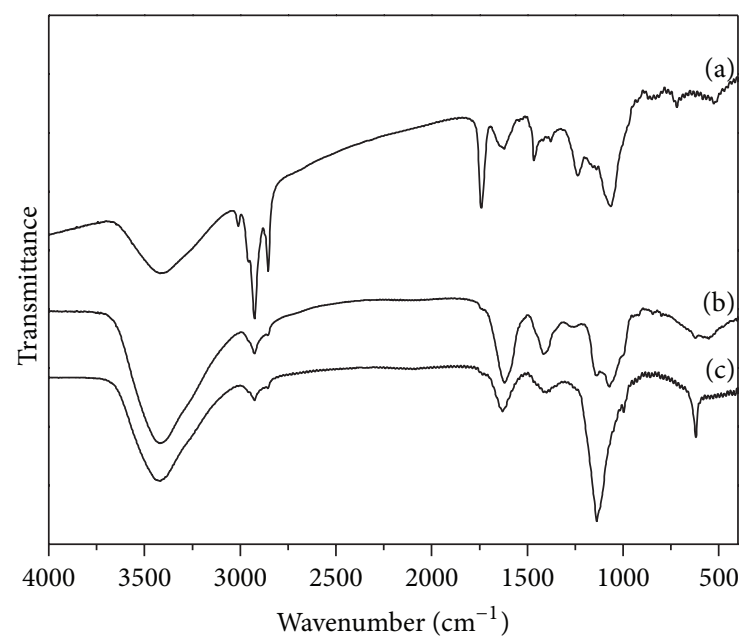

FIGURE 5: FT-IR spectra of (a) lecithin powder, (b) nanolecithin, and (c) lecithin-nano Ni/Fe.

by the interstices from the loading of bimetallic nanoparticles, which will profit the interaction between lecithinnano Ni/Fe and PCB77 during removal process. However, the inconspicuous difference represented the insufficiently loading amount of bimetallic nanoparticles which resulted in only a thin loading layer on the surface. This problem was possibly caused by the limited content of water for the microemulsion which was difficult to solve and probably affected the efficacy of the designed material in removing PCB77.

3.4. FT-IR Spectroscopy. The typical adsorption bands of various functional groups, respectively, for lecithin-nano Ni/Fe, nanolecithin, and lecithin powder were shown in Figure 5. From the spectrum of nanolecithin, it was observed that the notable absorbance peaks at $1620 \mathrm{~cm}^{-1}$ and $1418 \mathrm{~cm}^{-1}$ correspond to the asymmetrical and symmetrical stretching vibrations of $\mathrm{C}=\mathrm{O}$ groups [28]. The absorbance peaks at $2926 \mathrm{~cm}^{-1}$ and $2854 \mathrm{~cm}^{-1}$ were assigned to the asymmetrical and symmetrical stretching vibrations of $\mathrm{CH}_{2}$ groups. The broad band at $3419 \mathrm{~cm}^{-1}$ was attributed to the presence of water. The characteristic peaks at $1138 \mathrm{~cm}^{-1}$ and $1072 \mathrm{~cm}^{-1}$ were typical of $\mathrm{PO}_{2}^{-}$group which was attributed to the $\mathrm{P}=\mathrm{O}$ and $\mathrm{P}-\mathrm{O}$ stretches. Considering the neat composition of nanolecithin, all of the peaks above represented the character of lecithin. Although the spectrum of raw powder presented relative absorbance as aforementioned involving the peaks at

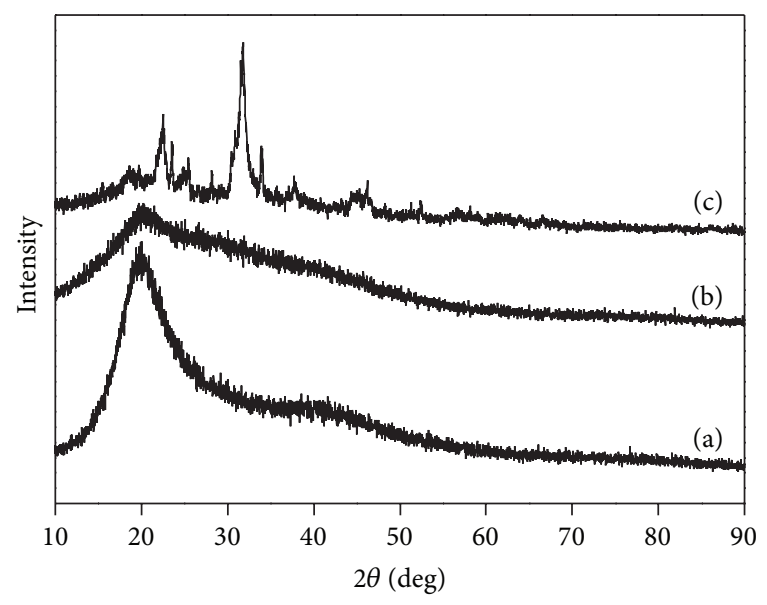

FIGURE 6: XRD patterns of (a) lecithin powder, (b) nanolecithin, and (c) lecithin-nano Ni/Fe.

$3406 \mathrm{~cm}^{-1}, 1236 \mathrm{~cm}^{-1}$, and $1065 \mathrm{~cm}^{-1}$, it had some differences in the regions in carbonyl stretching and methylene stretching. The asymmetrical and symmetrical stretches of $\mathrm{C}=\mathrm{O}$ groups had shifted to $1622 \mathrm{~cm}^{-1}$ and $1426 \mathrm{~cm}^{-1}$ with a spilt peak at $1739 \mathrm{~cm}^{-1}$, while those of $\mathrm{CH}_{2}$ groups intensified with spilt peaks at $2956 \mathrm{~cm}^{-1}$ and $3010 \mathrm{~cm}^{-1}$ [29]. It was predicted that the complex composition of the raw powder was purified through the deposition procedure, which correlated with the unitary adsorption bands of the nanocarrier with neat lecithin composition. The significant differences of spectra between lecithin-nano $\mathrm{Ni} / \mathrm{Fe}$ and nanolecithin revealed the notable influence of the loading of $\mathrm{Ni} / \mathrm{Fe}$ bimetal. Although the carbonyl stretches in lecithin-nano $\mathrm{Ni} / \mathrm{Fe}$ were similar to those in nanolecithin, the characteristic peaks of $\mathrm{PO}_{2}{ }^{-}$group in nanolecithin had been obscured in lecithin-nano $\mathrm{Ni} / \mathrm{Fe}$ due to the entity of a broad and intense band of the $\mathrm{C}-\mathrm{O}$ ester at $1138 \mathrm{~cm}^{-1}$. The presence of this new band was indicative of a change in the dielectric constant of the environment of the ester groups which was likely ascribed to the association of the lecithin carrier with the $\mathrm{Ni} / \mathrm{Fe}$ nanoparticle. It was signified by the FT-IR spectra that there was notable interaction of bimetal with lecithin which promoted the stable combination and effective loading of nanoparticles on the carrier.

3.5. XRD Patterns. The XRD patterns of all samples were displayed in Figure 6. The patterns of both nanolecithin and raw powder showed the same main diffraction peak appearing at $2 \theta$ value near $22^{\circ}$. While such a sole peak certainly corresponded to lecithin, it was more broad in the pattern of nanolecithin probably due to the broadening effect of the nanoscale. The loading of Ni/Fe nanoparticles afforded abundant diffraction peaks in the pattern of lecithin-nano Ni/Fe. Apart from the characteristic peak of lecithin, the main peaks at $2 \theta$ values of $28^{\circ}, 32^{\circ}, 38^{\circ}, 44^{\circ}, 52^{\circ}$, and $56^{\circ}$ were related to the metallic component. It was suggested that lecithinnano $\mathrm{Ni} / \mathrm{Fe}$ had significantly distinguishing structure from 


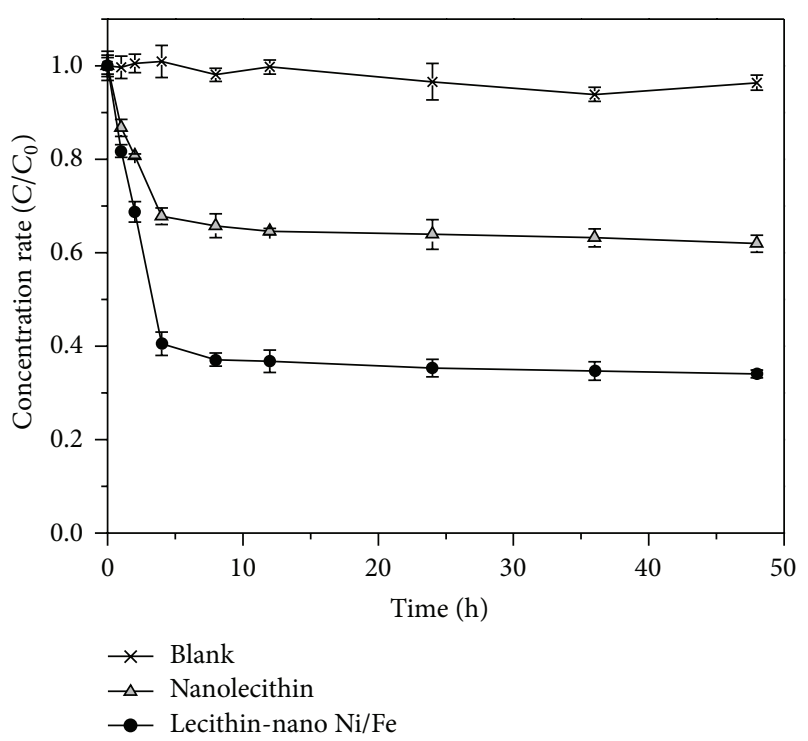

FIgURE 7: Removal of PCB77 in 50/50 (v/v) ethanol/water solution by lecithin-nano $\mathrm{Ni} / \mathrm{Fe}$ and nanolecithin.

the other samples through the effective loading of metallic particles. However, the characteristic peaks of metallic oxide, especially the intensity peak at $32^{\circ}$, exhibited the apparent oxidation extent of iron. The oxidation was possibly ascribed to the exposure of the active nanoparticles and will affect their reductive ability.

3.6. Removal of PCB77. The efficacy of different materials in removing PCB77 in 50/50 (v/v) ethanol/water solution was depicted in Figure 7. Approximately $67 \%$ of PCB77 was removed from the solution in $48 \mathrm{~h}$ by lecithin-nano Ni/Fe, while $39 \%$ of PCB77 was removed by nanolecithin. Since parallel experiment of laboratory blank indicated insignificant leakage, it was confirmed that nanolecithin had a certain activity to remove PCB77 as the blank carrier. The removal efficacy will further be improved by the $\mathrm{Ni} / \mathrm{Fe}$ nanoparticles loading on the carriers. Compared to nanolecithin, lecithinnano $\mathrm{Ni} / \mathrm{Fe}$ was able to decrease the concentration of PCB77 to a lower level in the same short period, which finally resulted in the lower residuary ratio. The synergistic effect of bimetal and lecithin apparently contributed to the rapid remov-al of PCB77 at the beginning. However, the favorable trend for the removal was slowed down after $4 \mathrm{~h}$ of experiment to achieve an almost constant concentration of PCB77. The limited loading content and difficultly escapable oxidation of the small bimetallic particles that had been revealed by part of the characterization presumably caused the rapid exhaustion of the activity. Although the function of the lecithin-nano $\mathrm{Ni} / \mathrm{Fe}$ still required to be improved, the rapid removal process at the beginning indicated the potentiality for the research of such an original combined material. Considering that the preliminary effectiveness of the combined material was proved in this work, the constitution should be optimized in the future for the better stabilization and loading efficiency of $\mathrm{Ni} / \mathrm{Fe}$ nanoparticles on the carrier, which will make the further researches about lecithin-nano Ni/Fe more attractive.

\section{Conclusion}

In this literature, lecithin carriers were loading $\mathrm{Ni} / \mathrm{Fe}$ nanoparticles through microemulsion method to generate a kind of combined material named lecithin-nano Ni/Fe. Lecithin participated in the composition of the microemulsion template that directed the synthesis of subtle $\mathrm{Ni} / \mathrm{Fe}$ nanoparticles and then formed the carrier through the deposition with the insite loading of bimetal. Besides the above role, lecithin, as an environmentally compatible biosurfactant, had a certain ability to accumulate organic contaminants, which was beneficial for the removal of the contaminants by $\mathrm{Ni} / \mathrm{Fe}$ nanoparticles. It was proved by the characterization that $\mathrm{Ni} / \mathrm{Fe}$ nanoparticles can be uniformly dispersed and closely combined with lecithin carrier which indicated the successful generation of lecithin-nano Ni/Fe. The synergistic effect of lecithin and $\mathrm{Ni} / \mathrm{Fe}$ nanoparticles was examined through the removal experiment of PCB77, while parallel test for the blank carrier of nanolecithin was operated as comparison. The preliminary functionality of lecithin-nano $\mathrm{Ni} / \mathrm{Fe}$ was supported by the more rapid removal of PCB77 at the beginning. Although it still required improvement owning to the exhaustion of activity in a relatively short period, lecithin-nano $\mathrm{Ni} / \mathrm{Fe}$ represented a beneficial attempt to acquire the combined material with the advantages of both the biosurfactant and the bimetal. The original synthetic method described in this paper was convenient with the stuff that was abundant, inexpensive, and relatively low toxic. It was prospective that this work can be the evocation for the development of innovative decontaminant and similar organic-inorganic combined material.

\section{Conflict of Interests}

The authors declare that there is no conflict of interests.

\section{Acknowledgment}

This work was supported by National Natural Science Foundation of China (21076140).

\section{References}

[1] J. H. Li, N. N. Zhao, X. Liu, and X. Y. Wu, "Achieving target of Stockholm Convention on PCBs elimination: Asia-Pacific case," in Proceedings of the International Conference on Management Science and Engineering (ICMSE '13), pp. 2147-2154, 2013.

[2] D. Megson, G. O'Sullivan, S. Comber et al., "Elucidating the structural properties that influence the persistence of PCBs in humans using the National Health and Nutrition Examination Survey (NHANES) dataset," Science of the Total Environment, vol. 461-462, pp. 99-107, 2013. 
[3] A. Ruus, I. A. Daae, and K. Hylland, "Accumulation of polychlorinated biphenyls from contaminated sediment by Atlantic cod (Gadus morhua): direct accumulation from resuspended sediment and dietary accumulation via the polychaete Nereis virens," Environmental Toxicology and Chemistry, vol. 31, no. 11, pp. 2472-2481, 2012.

[4] M. Olufsen and A. Arukwe, "Developmental effects related to angiogenesis and osteogenic differentiation in Salmon larvae continuously exposed to dioxin-like 3, 3', 4, 4'-tetrachlorobiphenyl (congener 77)," Aquatic Toxicology, vol. 105, no. 3-4, pp. 669-680, 2011.

[5] X. Liu, Z. Chen, Z. Chen, M. Megharaj, and R. Naidu, "Remediation of Direct Black $\mathrm{G}$ in wastewater using kaolin-supported bimetallic Fe/Ni nanoparticles," Chemical Engineering Journal, vol. 223, pp. 764-771, 2013.

[6] S. Mossa Hosseini, B. Ataie-Ashtiani, and M. Kholghi, "Nitrate reduction by nano-Fe/Cu particles in packed column," Desalination, vol. 276, no. 1-3, pp. 214-221, 2011.

[7] X. Wang, M. Zhu, H. Liu, J. Ma, and F. Li, "Modification of Pd-Fe nanoparticles for catalytic dechlorination of 2, 4dichlorophenol," Science of the Total Environment, vol. 449, pp. 157-167, 2013.

[8] E. M. Zahran, D. Bhattacharyya, and L. G. Bachas, "Reactivity of $\mathrm{Pd} / \mathrm{Fe}$ bimetallic nanotubes in dechlorination of coplanar polychlorinated biphenyls," Chemosphere, vol. 91, no. 2, pp. 165-171, 2013.

[9] S. K. Singh, A. K. Singh, K. Aranishi, and Q. Xu, "Noblemetal-free bimetallic nanoparticle-catalyzed selective hydrogen generation from hydrous hydrazine for chemical hydrogen storage," Journal of the American Chemical Society, vol. 133, no. 49, pp. 19638-19641, 2011.

[10] Y. Gao, H. Yang, X. Zhan, and L. Zhou, "Scavenging of BHCs and DDTs from soil by thermal desorption and solvent washing," Environmental Science and Pollution Research, vol. 20, no. 3, pp. 1482-1492, 2013.

[11] A. Singh and S. Cameotra, "Efficiency of lipopeptide biosurfactants in removal of petroleum hydrocarbons and heavy metals from contaminated soil," Environmental Science and Pollution Research, vol. 20, no. 10, pp. 7367-7376, 2013.

[12] E. Ceschia, J. R. Harjani, C. Liang et al., "Switchable anionic surfactants for the remediation of oil-contaminated sand by soil washing," RSC Advances, vol. 4, no. 9, pp. 4638-4645, 2014.

[13] N. Manickam, A. Bajaj, H. S. Saini, and R. Shanker, "Surfactant mediated enhanced biodegradation of hexachlorocyclohexane (HCH) isomers by Sphingomonas sp. NM05," Biodegradation, vol. 23, no. 5, pp. 673-682, 2012.

[14] S. Mohanty and S. Mukherji, "Surfactant aided biodegradation of NAPLs by Burkholderia multivorans: comparison between Triton X-100 and rhamnolipid JBR-515," Colloids and Surfaces B: Biointerfaces, vol. 102, pp. 644-652, 2013.

[15] X. Y. Qv and J. G. Jiang, "Toxicity evaluation of two typical surfactants to Dunaliella bardawil, an environmentally tolerant alga," Environmental Toxicology and Chemistry, vol. 32, no. 2, pp. 426-433, 2013.

[16] A. Azizullah, P. Richter, M. Jamil, and D. P. Häder, "Chronic toxicity of a laundry detergent to the freshwater flagellate Euglena gracilis," Ecotoxicology, vol. 21, no. 7, pp. 1957-1964, 2012.

[17] M. Song and A. R. Bielefeldt, "Toxicity and inhibition of bacterial growth by series of alkylphenol polyethoxylate nonionic surfactants," Journal of Hazardous Materials, vol. 219-220, pp. 127-132, 2012.
[18] P. Foley, A. Kermanshahi Pour, E. S. Beach, and J. B. Zimmerman, "Derivation and synthesis of renewable surfactants," Chemical Society Reviews, vol. 41, no. 4, pp. 1499-1518, 2012.

[19] E. Kaczorek, "Effect of external addition of rhamnolipids biosurfactant on the modification of gram positive and gram negative bacteria cell surfaces during biodegradation of hydrocarbon fuel contamination," Polish Journal of Environmental Studies, vol. 21, no. 4, pp. 901-909, 2012.

[20] X. Zhang, D. Xu, C. Zhu, T. Lundaa, and K. E. Scherr, "Isolation and identification of biosurfactant producing and crude oil degrading Pseudomonas aeruginosa strains," Chemical Engineering Journal, vol. 209, pp. 138-146, 2012.

[21] R. M. Jain, K. Mody, A. Mishra, and B. Jha, "Physicochemical characterization of biosurfactant and its potential to remove oil from soil and cotton cloth," Carbohydrate Polymers, vol. 89, no. 4, pp. 1110-1116, 2012.

[22] C. Zheng, M. Wang, Y. Wang, and Z. Huang, "Optimization of biosurfactant-mediated oil extraction from oil sludge," Bioresource Technology, vol. 110, pp. 338-342, 2012.

[23] D. M. Cabezas, R. Madoery, B. W. K. Diehl, and M. C. Tomás, "Emulsifying properties of different modified sunflower lecithins," Journal of the American Oil Chemists' Society, vol. 89, no. 2, pp. 355-361, 2012.

[24] A. Diab and M. Sandouka, "Effect of a phytogenic biosurfactant on the microbial community and on the bioremediation of highly oil-polluted desert soil," Journal of American Science, vol. 8, no. 1, pp. 544-550, 2012.

[25] F. Occulti, G. C. Roda, S. Berselli, and F. Fava, "Sustainable decontamination of an actual-site aged PCB-polluted soil through a biosurfactant-based washing followed by a photocatalytic treatment," Biotechnology and Bioengineering, vol. 99, no. 6, pp. 1525-1534, 2008.

[26] F. Sahle, H. Metz, J. Wohlrab, and R. H. Neubert, "Lecithinbased microemulsions for targeted delivery of ceramide AP into the stratum corneum: formulation, characterizations, and in vitro release and penetration studies," Pharmaceutical Research, vol. 30, no. 2, pp. 538-551, 2013.

[27] P. Fadda, M. Monduzzi, F. Caboi, S. Piras, and P. Lazzari, "Solid lipid nanoparticle preparation by a warm microemulsion based process: influence of microemulsion microstructure," International Journal of Pharmaceutics, vol. 446, no. 1-2, pp. 166175, 2013.

[28] C. Rosca, M. I. Popa, G. Lisa, and G. C. Chitanu, "Interaction of chitosan with natural or synthetic anionic polyelectrolytes. 1 . The chitosan-carboxymethylcellulose complex," Carbohydrate Polymers, vol. 62, no. 1, pp. 35-41, 2005.

[29] W. Jiang, J. A. Joens, D. D. Dionysiou, and K. E. O'Shea, “Optimization of photocatalytic performance of $\mathrm{TiO}_{2}$ coated glass microspheres using response surface methodology and the application for degradation of dimethyl phthalate," Journal of Photochemistry and Photobiology A: Chemistry, vol. 262, pp. 713, 2013. 

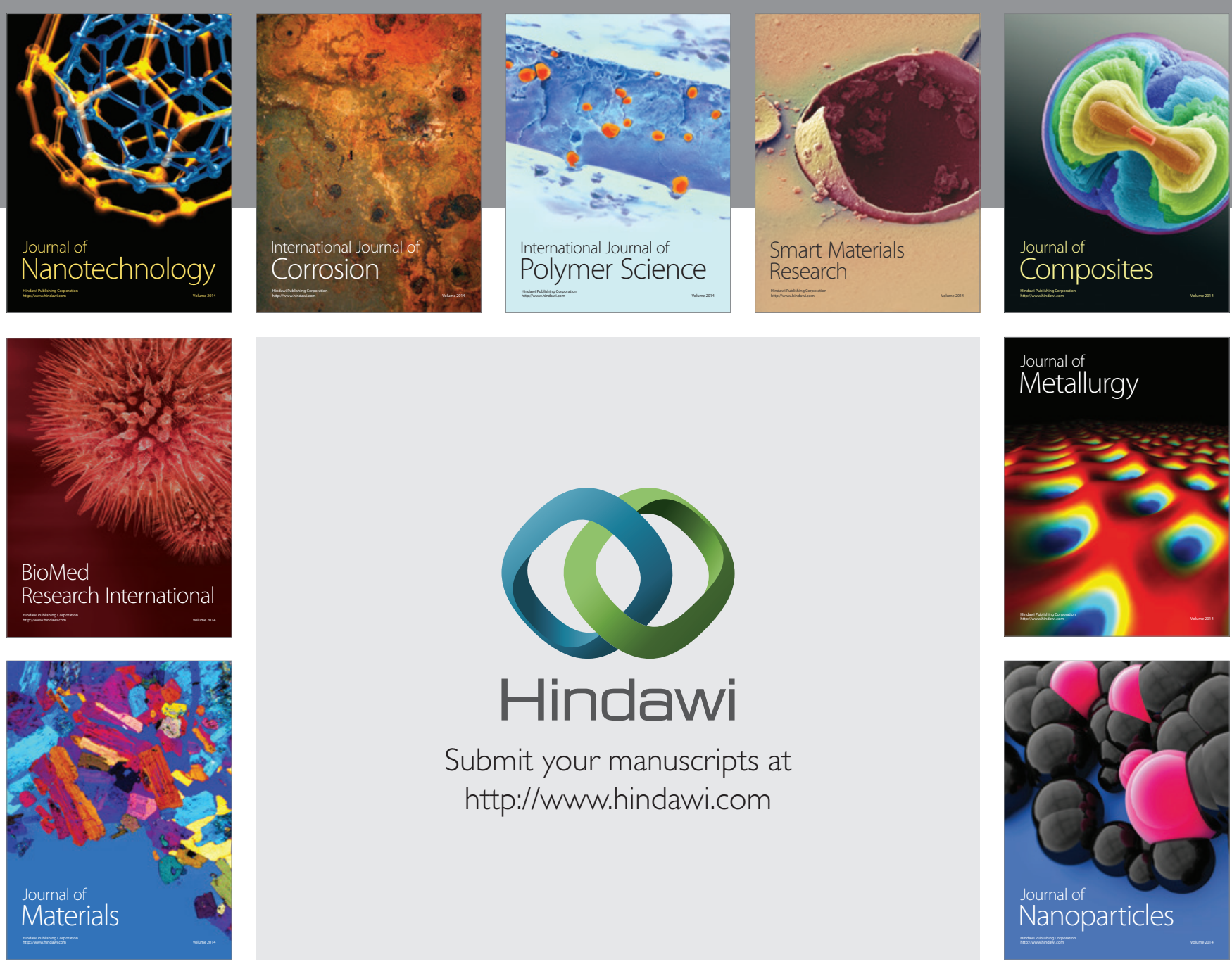

Submit your manuscripts at http://www.hindawi.com
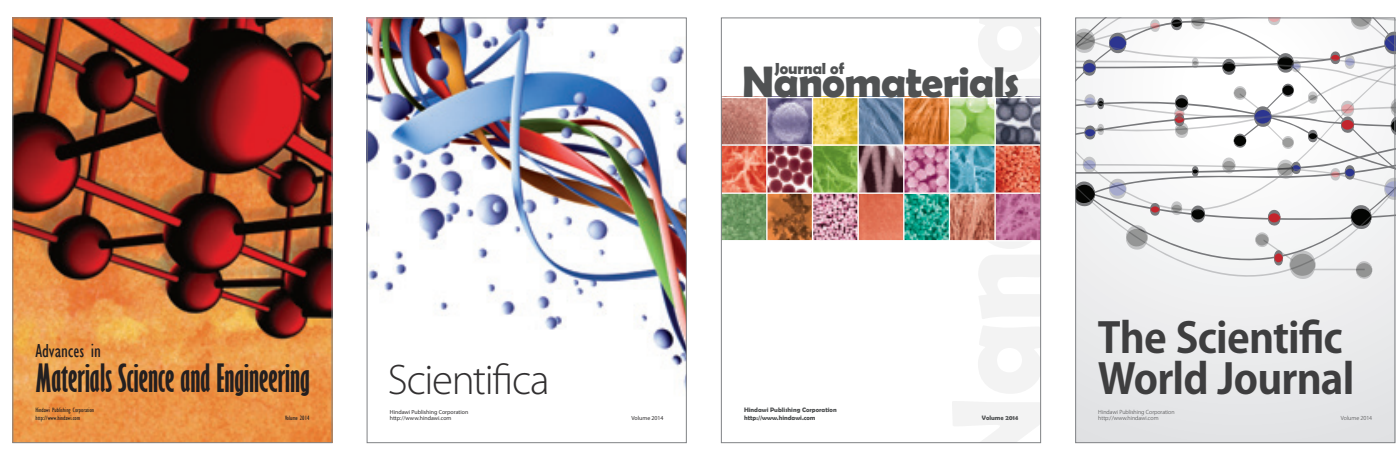

\section{The Scientific World Journal}
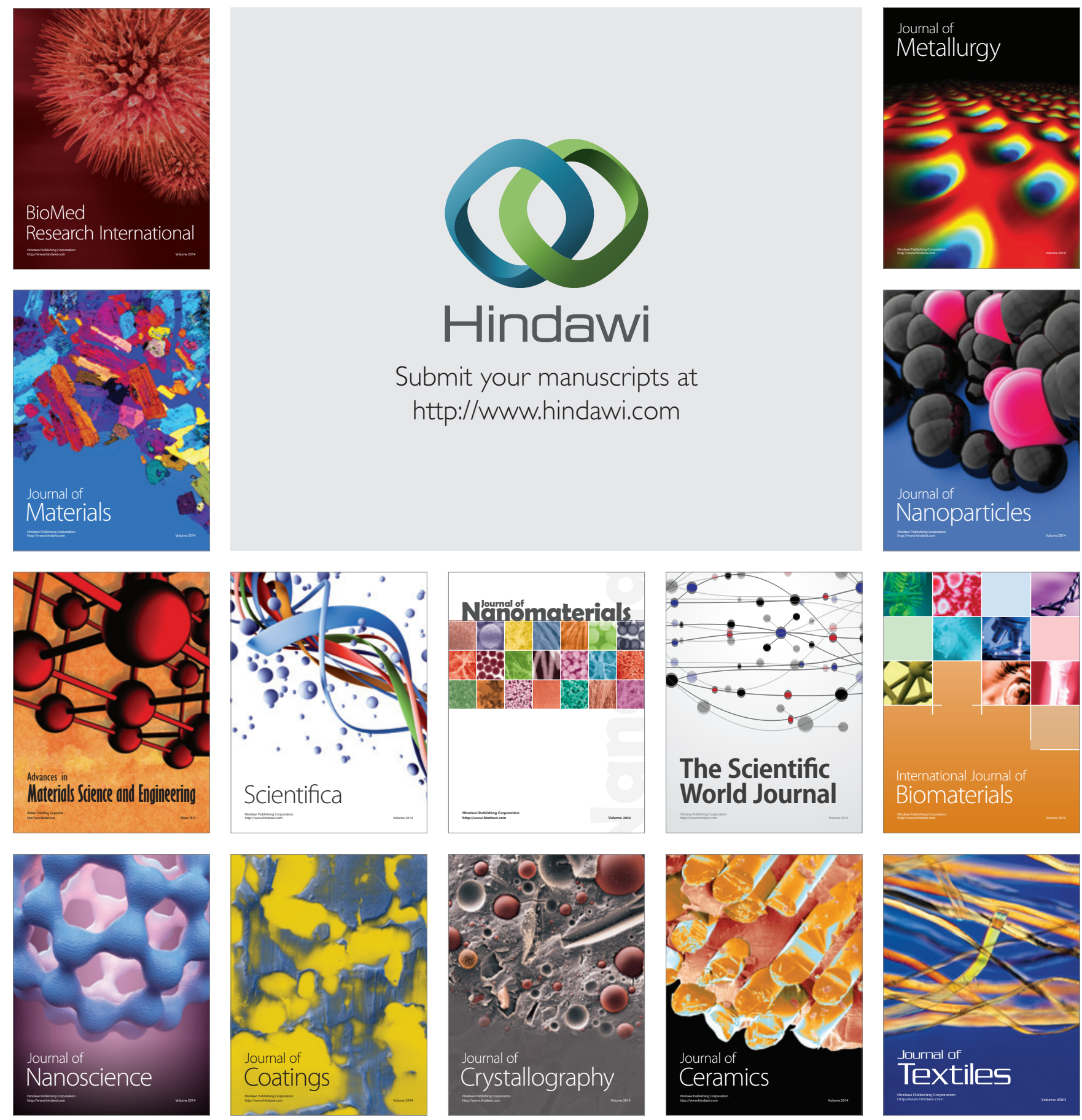